\title{
Henri IV «Padre delle buone lettere»?
}

\section{Bruno Petey-Girard}

\section{(2) OpenEdition}

\section{Journals}

\section{Édition électronique}

URL : http://journals.openedition.org/studifrancesi/6155

DOI : 10.4000/studifrancesi.6155

ISSN : 2427-5856

\section{Éditeur}

Rosenberg \& Sellier

\section{Édition imprimée}

Date de publication : 1 novembre 2010

Pagination : 442-455

ISSN : 0039-2944

\section{Référence électronique}

Bruno Petey-Girard, « Henri IV «Padre delle buone lettere»? », Studi Francesi [En ligne], 162 (LIV | III) |

2010, mis en ligne le 30 novembre 2015, consulté le 13 janvier 2021. URL : http://

journals.openedition.org/studifrancesi/6155; DOI : https://doi.org/10.4000/studifrancesi.6155

\section{(c) (i) (9)}

Studi Francesi è distribuita con Licenza Creative Commons Attribuzione - Non commerciale - Non opere derivate 4.0 Internazionale. 


\section{Henri IV «Padre delle buone lettere»?}

L'assassinat d'Henri IV en mai 1610 suggère nombre de célébrations posthumes du défunt roi de France. L'éloge de la protection accordée aux Lettres n'en constitue qu'un aspect très limité. Ainsi, et pour ne mentionner qu'un exemple, Jérôme de Bénévent, sur les trois-cent-cinquante-deux pages de son Discours des faicts berö̈ques de Henry le Grand, mentionne uniquement au détour d'une unique phrase l'action royale en faveur des collèges, des arts et du commerce ${ }^{1}$. Les gestes royaux en faveur des Lettres sont même le plus souvent passés sous silence ${ }^{2}$. C'est dire combien l'attention royale envers les arts et Lettres semble de peu d'importance au regard des autres titres de gloire du prince, principalement au regard de sa figure d'homme de guerre ayant ramené la paix dans le royaume.

Les milieux italiens ont été sensibles à une telle discrétion; l'éloge presque univoque du guerrier est jugé surprenant, surtout lorsqu'il - parce qu'il - prend place dans la bouche d'hommes de Lettres. Évoquant la célébration funèbre du roi dans l'espace littéraire que symbolise le Parnasse de ses Ragguagli où la nouvelle du régicide vient de parvenir et où se déroulent des actes di mestitia non piu vedut [i] in Parnaso doppo la morte del liberalissimo Ottaviano Augusto, Trajano Boccalini écrit en 1612²:

Piu di dugento lucubratissime orationi recitarono i Letterati di tutte le Accademie, delle Universitadi, e delle sette di Filosofi, e pur delle infinite vertudi di cosi grand Ré solo fù lodato il sopra humano valor militare di lui.

Une tension existe entre les éloges de lettrés à qui va manquer il Padre delle buone lettere, il Mecenate dei vertuosi et la teneur de la célébration: les Lettres ne louent pas leur protecteur, comme si le mécénat d'un roi, quelque unique qu'il soit, n'engendrait pas au royaume de France le portrait d'un prince-mécène. À la protection des Lettres (ne) répond (presque que) l'éloge du guerrier par les lettrés. Tout ici, on le voit, est affaire d'image et les remarques de Boccalini, si elles sont fondées, soulignent aussi - et ce point seul retiendra notre attention - que le roi de France est en droit de prétendre à un titre - Padre delle buone lettere - que la tradition nationale réservait depuis 1547 au seul François $1^{\mathrm{er} 4}$. Nous préciserons certains traits de

(1) J. DE BÉNÉvent, Discours des faicts herö̈ques de Henry le Grand, Paris, J. de Heuqueville, 1611, p. 293.

(2) Pour les oraisons funèbres du roi, cette rareté est soulignée par J. HENNEQUIN dans Henri IV dans ses oraisons funèbres ou la naissance d'une légende, Paris, Klincksieck, 1977, pp. 115-116 et dans «Les oraisons funèbres de Henri IV et Louis XIII, manifestations du mécénat», L'Âge d'or du mécénat, 1598-1661, éd. R. Mousnier et J. Mesnard, Paris, CNRS, 1985, pp. 329-349.
(3) T. Boccalini, De’ Ragguagli di Parnaso, Milan, G. Bidelli, 1614 (1 ${ }^{\text {ère }}$ éd. 1612), pp. 13-14.

(4) La formule est utilisée pour la première fois dans la proclamation de la mort du roi par le Parlement de Paris qui célèbre le «très hault, très puissant et très magnanime François, par la grace de Dieu Roy de France, très chrestien, premier de ce nom, prince clement, pere des arts et Sciences» (G. PARADIN, Histoire de nostre temps [...], Lyon, J. de Tournes, 1552, p. 533). Sa première occurrence 'littéraire' se lit sous la plume de Peletier du Mans; il 
l'image du prince face au monde de la culture lettrée au moment où l'Humanisme de la Renaissance voit son déclin se confirmer et où très rares sont ceux qui voient dans les Lettres le fondement tant de la société civile que de la vie politique. Si les Lettres ont leur place dans le royaume, celle-ci se trouve redéfinie par rapport à l'idéal qui poussait Budé à solliciter de son roi un appui pour les Muses 5 .

Au moment où Henri IV disparaît, il n'est plus le roi de Navarre que Jean de Sponde pouvait, en 1583, célébrer, s'adressant à lui, pour son soutien accordé aux Lettres:

De te primum ut loquar, iamdiu equidem mihi perspectum est, quanto studio studia haec nostra semper sis prosequutus: cuius uirtutis prope modum singularis (et enim Virtus, literas uirtutum officinam excolere) eadem tua mater haeredem te reliquit. Hinc uiri literati et eruditi suum quoque honorificum ordinem apud te plurimum sunt consequuti: hinc tua Academia illa Bearnensis efflorescit: quod amoris tui in literas argumentum est luculentissimum, ut nihil absurdum facere existimari debeam, qui literarum fautori literarium aliquid offeram ${ }^{6}$.

Rien ne manque dans ce portrait encore humaniste d'Henri de Navarre: amour des Lettres, attention attentive aux lettrés, le tout discrètement inscrit dans une dynamique familiale; le roi hérite de la reine sa mère une vertu rare, mais aussi, en raison de cet héritage, une vertu étroitement attachée à la personne royale. Rien ne manque, sauf, peut-être, l'image associée de prince savant qui aurait idéalement complété le tableau.

Les conditions qui accompagnent l'accession au trône de France du roi de $\mathrm{Na}$ varre à partir d'août 1589 ne favorisent guère la fixation de l'image spondienne. Plusieurs années de guerre voient le prince conquérir son royaume à la pointe de l'épée. L'image que les Lettres tracent alors de lui valorise un roi vainqueur des ennemis du trône, restaurateur de l'unité nationale. Mutatis mutandis, cette image n'est pas sans rappeler celle que les poètes traçaient d'Henri II au lendemain des victoires qui ouvraient son règne; une nuance cependant, et de taille: alors que l'on faisait souvent miroiter aux yeux du roi l'image glorieuse de son père François $1^{\text {er }}$, amateur et protecteur des Lettres, nul ne suggère qu'Henri IV ait besoin d'un quelconque modèle en matière de soutien des Lettres. À en croire certains textes où son image se fixe, il serait déjà, fût-ce à sa manière, un prince protecteur des Lettres et les lettrés peuvent se tourner vers lui comme source d'une reconnaissance qui justifie l'existence même de leurs œuvres.

Jean Godard publie ainsi en 1594 ses Trophées de Henry Quatriesme, très chrestien et très victorieux Roy de France et de Navarre; il célèbre un roi qui doit «Fermer du Dieu

offre en effet à François $1^{\text {er }}$ sa version d'Homère et l'interpelle: «Et toy qui es des lettres le vray pere...» (Les Euvres poetiques, Paris, Vascosan et Corrozet, $\left.1547, \mathrm{f} .84 \mathrm{v}^{\circ}\right)$ Les vers de Peletier ne sont publiés qu'à la fin de l'année 1547, soit quelques mois après la disparition du roi.

(5) Sur cet idéal, voir la correspondance de Budé ainsi que les deux ouvrages parus en 1532: De studio literarum et De philologia; sur son déclin dans les années 1600-1640, voir H. MERLin-Kajman, L'excentricité académique. Littérature, institution, société, Paris, Les Belles Lettres, 2001.

(6) J. DE SPONDE, Homeri quae extant omnia [...], Bale, E. Bischoff, 1583. Peut-être faut-il voir dans cette académie le collège où Sponde a fait ses études avec le soutien matériel de la reine Jeanne puis du roi Henri, collègue qui est en 1583 érigé en une université que le roi de Navarre qualifie alors de «regia nostra Academia»; voir M. LourdeRoCHEBLAYE, Les anciennes académies protestantes, B.S.H P F., $3^{\mathrm{e}}$ année, 1855 , pp. 280-281; P. DE FELICE, Les lois collégiales de l'Académie de Béarn (1569-1580), Paris, Imp. Nat., 1880, pp. 5-9, 1415; J. Coudirolle, Etude sur l'Académie d'Orthez, fin du XVI et commencement du XVII siècle, Paris, A. Derenne, 1885 , pp. 73-75; E. Droz, Les années d'études de Jean et Henry de Sponde, B.H.R., IX [1947], pp. 142-143. Mais existe également une «petite Academie» fondée par Henri à quoi d'Aubigné fait allusion dans une des ses «Lettres sur diverses sciences», Euvres, éd. H. Weber, Paris, Gallimard, 1969, p. 848. 
Janus le temple à double porte», qui a su «rendre sa couronne et son sceptre asseuré», qui «remet sus l'Estat» ${ }^{7}$. Loin d'exploiter l'image traditionnelle d'Alexandre en quête d'un poète qui, tel Homère immortalisant Achille par son chant, fixerait sa geste - en aurait jailli une image royale de protection des Lettres -, il inverse les désirs:

Poëtes, qui chantez mon Prince et mon Seigneur

Que ce vous est de bien, et de gloire et d'honneur,

De voir son nom au front de vostre poësie. ${ }^{8}$

Aucun appel n'est lancé au prince afin qu'il favorise sa propre célébration en témoignant sa faveur aux lettrés; la grandeur du prince rejaillit sur la grandeur de la poésie qui le loue. C'est que l'image royale n'a pas besoin d'être suscitée par quelques souhaits. Dans la seconde édition des Trophées, Godard trace le portrait d'un prince

plus liberal envers les gens doctes, et gens de bien lettrez que Trajan envers le Philosophe Dion [...], que Gratien envers Ausone [...], qu'Alexandre envers Aristote, auquel pour ses livres de la nature des animaux lui donna huict cens talens.

Le roi semble réaliser les attentes des hommes de Lettres sans qu'il soit besoin de l'y pousser. Cet éloge se retrouve sous la plume de Gabriel Chappuys qui établit pour sa part un parallèle entre François $1^{\text {er }}$ et Henri IV

Le monde a grande raison de louer singulierement François I. Roy de France [...], à cause de l'esprit qu'il a donné aux bonnes lettres quasi mortes et ensevelies lors, en son Royaume: mettant es Escoles de Paris [...] excellents professeurs en toutes sortes de sciences, que nostre prince incomparable, nonobstant les grands desordres des guerres passées, n'a laissé d'entretenir, depuis que par les armes, et par la grace celeste, il s'est veu recognu de ses subjects. ${ }^{10}$

Les Lettres publient donc une image déjà formée.

Ces gestes royaux de protection ne sont pas le support essentiel de la célébration usuelle du prince, mais le roi demeure celui vers qui nombre de lettrés tournent leur regard. Godard se place ainsi dans une dynamique de reconnaissance de son œuvre par le prince: le sonnet qui clôt les Trophées est adressé à Pierre Forget qui, tel Mécène présentant Virgile à Auguste, pourrait «conduire sa Muse vers le roi»; le commentaire de Claude Le Brun qui accompagne la seconde édition précise que le poète

eust en vain si laborieusement travaillé pour la célébration des louanges de sa Majesté [...] si la dicte Majesté par quelque advertissement n'en avoit cognoissance. ${ }^{11}$

Sans prendre davantage les traits d'un possible protecteur des Lettres, le roi n'en est pas moins l'ultime instance de reconnaissance du poète qui le loue. Pierre Matthieu confirme cette tendance et écrit en 1610:

Les choses ne sont considerées en ceste Cour, si vostre parole ne les approuve et vostre liberalité n'en confirme l'approbation. ${ }^{12}$

(7) J. Godard, Les Trophées de Henry Quatriesme [...], Lyon, P. Dauphin, 1594, pp. 4, 14 et 16.

(8) Ibid., p. 27.

(9) J. GODARD, L'Oracle ou chant de Protée, où sont predictes les glorieuses victoires de Henry IIII [...] ensemble les Trophées [...]. Avec le commentaire de Claude Le Brun, Lyon, Ancelin, 1594, f. *** ${ }^{\circ}$.
(10) G. Chappuys, épître en tête de sa version de G. Botero, Raison et gouvernement d'estat [...], Paris, G. Chaudière, 1599, f. [è] $]^{6} \mathrm{r}^{\circ}$.

(11) J. GodarD, L'Oracle ou chant de Protée, pp. 133-134.

(12) P. Matthieu, Histoire de Louis XI, Paris, P. Mettayer, 1610, f. [à] $]^{3} \mathrm{r}^{\circ}$, dédicace «Au Roy». 
Le roi contribue donc à la réputation des hommes de Lettres dans les cercles de la cour. C'est peut-être pourquoi, dans les étrennes qu'il lui adresse en 1602, Nicolas Rapin lui soumet son projet de restauration de la poésie mesurée:

O grand Roy, si tu veulx d'un favorable acueil

Nostre effort recevoir, é l'aprouver de l'œil,

Nous vaincrons l'ignorance. ${ }^{13}$

Claude Garnier soumet aussi sa plume à la volonté royale:

Comme un bon Prince enfle moy le courage,

Sinon de biens, au moins d'un bon visage. ${ }^{14}$

Une telle requête évoque celles que les poètes des Valois avaient écrites; Garnier en a conscience et place son écriture dans le sillage de celle de Ronsard:

J'iray vers toy pour te rendre en mon art

Tel que Henry fut rendu par Ronsard:

Henry second bien heureus d'influence

Qui vit surgir les Muses en la France. ${ }^{15}$

Si le parallèle met avant tout en scène la possible filiation des deux poètes, il ne manque pas de pertinence quant à l'image des deux princes, l'un comme l'autre prioritairement perçus comme rois de guerre. Rappeler à Henri IV que le règne de son prédécesseur a vu «surgir les Muses en la France» indique subtilement que son propre règne pourrait être celui d'un autre renouveau des Lettres. Tout en soulignant que le poète est celui qui la crée, Garnier ne joue cependant pas sur l'image du prince dont le lecteur ne sait pas précisément ce qu'elle est. Le roi, maître supérieur de la faveur, est sous sa plume le seul à pouvoir reconnaître le travail accompli ou à venir. Si cependant l'approbation royale est sentie comme nécessaire, elle n'est plus rattachée à un intérêt particulier du prince pour les Lettres: le prince n'est pas peint en amateur humaniste des Lettres et nul ne cherche à faire de lui un amateur des Lettres. L'amour des Lettres semble même se replier hors de la sphère royale. Si son œuvre est introduite auprès du roi, Godard ne célèbrera que Forget comme amateur et protecteur des Lettres:

Je diray dans mes vers encore apres cent ans,

Que les Muses Forget aima tant de son temps,

Qu'il estoit leur appuy, leur support, et leur gloire. ${ }^{16}$

Le profil humaniste du prince se défait. En regard, la figure d'un mécène qui assurerait le lien entre un prince distant et les Lettres se dessine. Il semble même parfois que le(s) ministre(s) seul(s) assure(nt) la visibilité des Lettres. Ainsi, J. de Rougevalet, adressant à Sully les Euvres poetiques de Passerat en 1606, évoque un ministre tout

(13) N. RapIN, «Ode mezurée, é rimée présantée pour estrenne au Roy», Euvres, éd. P. BRunEL, t. 1, Genève, Droz, 1982, p. 671.

(14) C. GARNIER, «Discours Au Roy», en tête des Royales Couches, ou les Naissances de Monsieur le Dauphin et de Madame [...], Paris, A. L'Angelier, 1604, f. Aiijv ${ }^{\circ}$ Cf. la dédicace adressée au roi par
Salomon Certon en tête de sa traduction de L'Odyssée (Paris, A. L'Angelier, $1606, \mathrm{f}$. àiijv ${ }^{\circ}-[\grave{a}]^{4} \mathrm{r}^{\circ}$ ) où Certon subordonne son possible éloge du roi à sa «grace» et à sa «douce faveur».

(15) Ibid., f. $[A]^{5} \mathrm{r}^{\circ}$.

(16) J. GODARD, Les Trophées de Henry Quatriesme..., loc. cit. 
au service d'un prince dont la figure n'apparaît que lointaine; certes, le roi a 'élu' Sully «pour l'assister au restablissement de l'Etat», mais en Sully seul - le ministre est sur ce point indépendant de son prince - il loue

le soin qu'[il a] de faire passer aux suivants la mémoire de nostre heureux siecle signalé, tant par les superbes bastiments et autres marques muettes de nostre felicité et repos, qui se voyent çà et là, que par la solicitude qu'[il prend] en la recherche des hommes sçavans et publication de leurs œuvres. ${ }^{17}$

Ouvrier de la gloire royale - et non agent du roi en la matière -, Sully l'est aussi de la vie des œuvres; aucun lien cependant n'unit explicitement gloire du prince et vitalité des Lettres. Pour encore tournés qu'ils soient vers le prince, les lettrés rendent aussi compte de la nature nouvelle des relations qu'ils entretiennent avec lui. Leurs déclarations d'ailleurs ne signifient pas ce qu'est intimement un roi dont les Lettres soulignent peu les goûts en matière de Lettres et ce sans que pointe jamais à ce sujet le moindre regret ou le moindre désir de faire évoluer la situation. Le portrait royal qui se trace est sans doute celui que le prince accepte de voir tracer de lui mais surtout celui qu'il est possible de tracer de lui. L'image de la protection royale des Lettres évolue en fonction de données que l'on peut penser, au regard de la manière dont elle s'est forgée au siècle précédent, liées en partie au goût personnel du roi. Mais cette évolution traduit aussi certaines attentes nouvelles, et peut-être moins ambitieuses - ou ambitieuses dans leur ordre propre -, des lettrés et, avec elles, la transformation de l'image royale de protection des Lettres telle qu'elle avait pu s'incarner en François $1^{\text {er }}$.

Malgré ces changements profonds, le ton général n'est pas nostalgique ${ }^{18}$ et le roi demeure un dédicataire à qui l'on adresse des textes de toute nature sans regretter ce qui n'est plus. Dans les dédicaces, l'image de protection des Lettres ne se lit que rarement - et la rareté de l'image, loin de faire son prix, assure surtout son manque de visibilité, sinon de pertinence. L'éloge du roi de guerre victorieux se module progressivement et, même s'il reste un élément central, fait une large place, après la signature de la Paix de Vervins et de l'Édit de Nantes en 1598, à l'image du prince pacificateur qui a su assurer la stabilité et l'unité de l'État ${ }^{19}$ : Henri IV a créé une situation favorable à l'épanouissement des Lettres et ne les protège peut-être que par ce biais. Ce sentiment est sensible dès 1599 dans les premières pages des Muses françoises ralliées ${ }^{20}$ mais il revient à l'auteur de L'Astrée, Honoré d'Urfé, de le résumer en 1610; il offre alors son roman au roi et écrit:

Recevez la [i. e. L'Astrée] donc (SIRE) non comme une simple Bergere: mais comme une œuvre de vos mains, car veritablement on vous en peut dire l'Autheur: puisque c'est un enfant que la paix a fait naistre, et que c'est à vostre Majesté à qui toute l'Europe doit son repos, et sa tranquillité. ${ }^{21}$

(17) J. De Rougevalet, dans le Recueil des oeuvres poetiques de Jean Passerat, Paris, C. Morel, 1606, f. àij.

(18) La nostalgie semble une affaire de génération et si Desportes regrette le passé, il le fait l'année de sa mort; voir Epitaphe de J. Passerat dans Recueil des auvres poetiques de J. Passerat, Paris, C. Morel, 1606, p. [465].

(19) Voir C. VIVANTI, Guerre civile et paix religieuse dans la France d'Henri IV, Paris, Desjon- quères, 2006 et M. BIDEAux, «Quand commence le XVII ${ }^{\mathrm{e}}$ siècle?», D'un siècle à l'autre. Littérature et société de 1590 à 1610, Fasano-Paris, Schena editore-PUPS, 2001, pp. 58-62.

(20) Les Muses françoises ralliées de diverses pars, Paris, M. Guillemot, 1599, épîtres liminaires.

(21) H. D’URFÉ, dédicace (1610) de la seconde partie de L'Astrée, lue dans l'édition de Paris, A. Courbé, 1633. 
Par le jeu des homonymes, l'héroïne romanesque rejoint la fille de Zeus et de Thémis dont le retour est associé au retour de l'Âge d'or, grand thème de la célébration monarchique depuis le siècle précédent. Le roi auteur de la paix est l'auteur des œuvres qui naissent dans ce climat propice. Il n'en vient cependant pas à habiter un quelconque Parnasse. Le frontispice gravé par Léonard Gaultier du Parnasse des plus excellens Poetes de ce temps qui paraît en 1607 en témoigne exemplairement ${ }^{22}$ : Apollon et des Muses, directement dérivés des figures peintes par Raphaël un siècle auparavant au mur de la Chambre de la Signature, trônent sur le mont qui leur est consacré tandis que Pégase prend son élan en arrière-plan; de part et d'autre du cartouche où s'inscrit le titre du volume, Homère et Virgile se répondent. Le roi n'est présent que par le privilège qu'il a accordé pour cette publication: «Avec privilege du Roy», lit-on sous la représentation d'Homère. Mais il ne s'agit que d'un signe administratif de sa présence et aucun autre souverain qu'Apollon ne prend place sur le Parnasse qui attire le regard du lecteur. La figure du prince est à la fois centrale et marginale tandis que les lettrés, vrais maîtres des images du prince qu'ils fabriquent, ne font pas de la protection des Lettres une facette majeure de l'image royale.

Ce nouveau positionnement du roi face au monde des Lettres se voit dans une gravure de Jaspar Isac qui sert en 1614 de frontispice à la traduction française des Images de Philostrate par Blaise de Vigenère ${ }^{23}$. Conçue du vivant du roi ${ }^{24}$, cette somptueuse édition illustrée s'ancre sur un travail que Vigenère avait entrepris entre 1575 et 1578. Henri III avait demandé à Amyot de traduire en français les Images de Philostrate; l'évêque d'Auxerre s'y était refusé, considérant cette traduction impossible; finalement, Vigenère réalisa le travail. Il n'est pas neutre que la dimension royale de ce travail soit dévoilée sous le règne d'Henri IV; rien en effet ne laisse entendre, dans les éditions parues du vivant de l'auteur ou dans les éditions posthumes parues avant celle de 1614, que le roi ait un lien quelconque avec cette entreprise; rien ne le signale non plus explicitement en 1614; mais Antoine Du Verdier, dans l'édition de 1604 de sa Prosopographie, a révélé les origines de la traduction, la requête d'Henri III à Amyot et la réponse de Vigenère ${ }^{25}$. Entreprise royale donc, la traduction est en 1614 placée sous un regard royal. Le frontispice architectural donne à voir, entre les deux ailes-galeries d'un bâtiment en U, une représentation complète du Parnasse: Apollon, lyre en main, est accompagné des Muses; derrière lui se dresse le double mont de l'Hélicon; Pégase frappe du pied le sol d'où jaillit la source Hippocrène. L'espace ainsi figuré - une manière de temple des Muses - est séparé du monde extérieur par les bâtiments qui l'entourent: le Parnasse est un espace peut-être privilégié, mais surtout distinct du monde commun. À la baie centrale du bâtiment couvert d'une vaste coupole qui ferme la perspective formée par les galeries, le roi dont l'image est disproportionnée jette son regard sur ce Parnasse ${ }^{26}$. Le roi ne participe pas de ce monde

(22) Le Parnasse des plus excellens Poetes de ce temps, Paris, M. Guillemot, 1607.

(23) Les Images ou tableaux de platte peinture des deux Philostrates sophistes Grecs [...]. Mis en François par Blaise de Vigenere [...], Paris, Vve A. L'Angelier, 1614.

(24) Voir l'avertissement au lecteur, reproduit dans J. BAlSAMO - M. SimONin, Abel L'Angelier et Françoise de Louvain (1574-1620), Genève, Droz, 2002, p. 407.

(25) Ant. Du Verdier, Prosopographie, ou Description des hommes illustres et autres [...], Lyon, A. Gryphe, vol. III, pp. 2572-2573.

(26) Il paraît difficile de suivre F. Graziani qui voit dans cette image un portrait de Vigenère
(«Introduction» de son édition des Images, Paris, Champion, 1995, p. lxx); elle ne ressemble en effet en rien au portrait du traducteur que L'Angelier offrait dans l'édition des Decades de Tite-Live en 1606; la silhouette générale, la coiffure sont celles des portraits du roi. Voir, sur ce frontispice et sur la présence royale qui s'y met en scène, M. FumAROLI, «La Galleria de Marino et la Galerie Farnèse: épigrammes et œuvres d'art profanes vers 1600», L'École du silence. Le sentiment des images au XVII siècle, Paris, Flammarion, 1994, pp. 41-42; R. CRESCENZO, Peintures d'instruction. La postérité littéraire des Images de Philostrate en France, de Blaise de Vigenère à l'époque classique, Genève, Droz, 1999, pp. 161-165. 
parnassien, mais il semble le considérer tout en le dominant de sa présence; il n'est pas suggéré qu'il puisse être l'Apollon de ce temple des Muses pourtant placé sous ses auspices. Deux tableaux placés dans les angles inférieurs du frontispice représentent Paris et Athènes et rendent sensible le rôle culturel de la capitale française. À l'aplomb de la coupole, entouré de la devise «VT SOL», le soleil répand ses rayons bienfaisants, chassant d'épaisses nuées; il n'est pas douteux que ce soleil fait écho à l'influence royale en toute matière. En 1604, Certon évoque le

Grand Roy [...]

Dont la vertu remit [s] on redoutable état

En son lustre premier, dont le soleil rabat

Noz brouillars nuageux, et de rayons nouveaux

Enfantez de la paix rend vigoureux et beaux

Tous les coins de la France. ${ }^{27}$

Le portrait du frontispice de 1614 semble ainsi dire du prince tout qui peut en être dit en matière de protection des Lettres. Même au seuil d'un livre, le roi ne participe pas de l'univers allégorique où se détache son image. Mais sa présence, à la fois impérative et distante, patronne le monde des Lettres; tel le soleil, le roi répand des rayons qui rendent possible la vie des Lettres cantonnée dans un espace prestigieux et clos qui n'est pas celui du pouvoir, tout en en dépendant peut-être matériellement. Ainsi qu'on le lit sous la plume de Pierre Matthieu alors qu'il évoque la formation d'un jeune prince,

Sans espouser les arts, ny sans les ignorer

C'est estre assez sçavant que de les honorer. ${ }^{28}$

L'image de prince savant et aimant les Lettres se transforme et fait place à celle d'un prince savant selon les besoins de sa fonction. Face aux savoirs lettrés, l'image $\mathrm{du}$ roi sera celle, distante mais avec une intelligence toute politique, d'un roi 'honorant' les Lettres. Ce glissement est fondamental: il permet d'entrevoir le passage d'une image individuelle du prince protecteur des Lettres à une image royale et générique de la protection des Lettres.

Il semble qu'il ne faille pas uniquement voir dans ces images une fantaisie d'hommes de Lettres que le pouvoir se contenterait de ne pas contredire. En effet, l'évolution que connaît l'ornementation des palais royaux fait écho à ces figurations qui donnent leur place aux Lettres et aux arts dans les soins d'un prince.

La dimension avant tout militaire de l'image royale trouve un écho théorique dans les propositions qu'Antoine de Laval fait à Sully vers 1605 en vue de définir ce que sont les Peintures convenables aux basiliques et palais du Roy et mesme [i. e. surtout] à sa gallerie du Louvre ${ }^{29}$. L'auteur est attaché à la culture lettrée; il déplore ainsi

(27) L'Odyssée d'Homere, f. àijr ${ }^{\circ}$. L'image du roi soleil de son royaume est d'une relative banalité: elle avait été utilisée pour Henri III; elle le sera après Henri IV pour ses fils et petit-fils (avec le succès que l'on sait). Voir A. Joly, Le Roi-Soleil, histoire d'une image, «Revue de l'Histoire de Versailles», oct.-déc. 1936, pp. 213-235.

(28) P. MatThIEU, Histoire de Louys XI, f. [B] ${ }^{4} \mathrm{r}^{\circ}$.

(29) Ant. DE Laval, Traicté des peintures convenables aux basiliques et palais du Roy et mesme à sa gallerie du Louvre, à la suite des Desseins de professions nobles et publiques, Paris, A. L'Angelier, 1605; voir J. ThuillieR, «Peinture et politique; une théorie de la galerie royale sous Henri IV», Études d'art français offertes à Charles Sterling, éd. A. CHÂTELET et N. Reynaud, Paris, PUF, 1975, pp. 175-205 et J.-M. Chatelain, Morale de l'bistoire, immortalité de la Fable: un projet de galerie royale à l'âge du Gallicanisme, «Revue d'histoire moderne et contemporaine», n. 39, 3 [juillet-sept. 1992], pp. 449-464. 
le «retour de la barbarie» qui a accompagné les guerres, et fait sien le reproche du Tasse à la France, «que les Nobles abandonnent les Lettres, les laissent manier au petit peuple» ${ }^{30}$. Lorsqu'il s'agit cependant de donner une image du roi dans ses palais, aucune place n'est accordée à la représentation d'une éventuelle intervention royale dans le monde des Lettres. La chose ne surprend pas: les Valois n'avaient pas non plus accepté que leur iconographie soit associée au soutien dont ils pouvaient favoriser les Lettres et les images du prince protecteur des Lettres étaient de ce fait restées dans les livres ${ }^{31}$. Ce refus traditionnel, Laval le radicalise en refusant toute décoration allégorique dans les palais royaux. Certaines des images principalement véhiculées par les Lettres - telle celle du Parnasse que d'ailleurs il ne mentionne pas - devraient être évacuées au profit de l'exclusive célébration historique du souverain. L'histoire est pour Laval le sujet par excellence convenable à la grandeur d'un roi et cette histoire n'est en rien une histoire de la culture lettrée - si l'histoire des Lettres se constitue alors, elle reste confinée dans les livres ${ }^{32}$-; l'histoire que Laval souhaite voir mise en image est celle qui valorise, et presque exclusivement, la geste héroïque des princes. Quant aux portraits du roi qui doivent être présentés aux yeux des visiteurs, ils se doivent aussi d'exalter sa force militaire et ne laissent pas la moindre place à l'image d'un roi qui «fait fleurir les lettres». Sans doute un tel programme ne sera-t-il pas appliqué à la lettre dans les grands décors monarchiques du règne aujourd'hui disparus: au plafond de la Petite Galerie du Louvre se voyait ainsi une Gigantomachie où Jupiter, sous les traits d'Henri IV, foudroyait les Géants, images de la Ligue ${ }^{33}$. Au centre du plafond de la Galerie de Diane à Fontainebleau, le roi était représenté conduisant un quadrige triomphal tiré par des lions qu'entouraient des figures des vertus morales ${ }^{34}$. Si l'image du roi protecteur des Lettres se module dans les textes, elle reste dans un premier temps aussi absente qu'au $\mathrm{XVI}^{\mathrm{e}}$ siècle dans les décorations qui, de manière toujours plus sensible, exaltent la gloire de la personne royale en lui donnant une forme visible. Dans les dernières années du règne cependant, la très riche décoration sculptée qui couvre la façade sur Seine de la grande Galerie construite au cours du règne pour relier le Louvre aux Tuileries semble bien proposer, et pour la première fois, une sorte d'exposé emblématique et symbolique des divers centres d'intérêt du prince, sinon exactement des espaces relevant spécifiquement de sa protection. De ce vaste ensemble - un bâtiment long de quelque 450 mètres -, seule est conservée la partie est dont la construction est, au dire de la tradition, confiée à l'architecte Louis Métezeau ${ }^{35}$. Il est impossible d'affirmer que l'ensemble de la décoration a été envisagé sous sa forme finale dès le milieu des années 1590; par ailleurs, on ne connaît pas avec certitude les dates de réalisation de ce programme entamé dès janvier 1595 . Toujours est-il que, côté sud, les reliefs du rez-de-chaussée, élevé avant 1599 - ils portent le

(30) Ant. DE Laval, Desseins de professions nobles et publiques, f. $34 \mathrm{v}^{\circ}-36 \mathrm{v}^{\circ}$.

(31) Sur ce point, nous nous permettons de renvoyer à notre ouvrage, Le Sceptre et la plume. Images (littéraires) du prince protecteur des Lettres de la Renaissance au Grand Siècle, Genève, Droz, 2010.

(32) Voir E. Morgat-Longuet, Clio au Parnasse. Naissance de l'«bistoire littéraire» française aux XVI' et XVII siècles, Paris, Champion, 2006.

(33) Voir D. CoRDELlier, «Le décor de la petite Galerie sous Henri IV», La Galerie d'Apollon au palais du Louvre, dir. G. Bresc-Bautier, Paris, Gallimard-Musée du Louvre, 2004, pp. 32-38.

(34) Voir, sur ces deux portraits allégoriques du roi et leurs contextes iconographiques, F. BARDON,
Le portrait mythologique à la cour de France sous Henri IV et Louis XIII. Mythologie et politique, Paris, Picard, 1974 , p. 74 et suiv. On notera au passage que le parti adopté lors de la décoration picturale de la Petite Galerie aussi bien que de la Galerie de Diane constitue un moyen terme entre la théorie défendue par Laval et la tradition de décoration allégorico-mythologique chère aux Valois: l'histoire occupe largement les murs - portraits des rois de France au Louvre et victoires du roi à Fontainebleau - tandis que les voutes reçoivent un décor à perspectives plafonnantes centré sur le mythe.

(35) La partie ouest, attribuée à Jacques II Androuet du Cerceau, fut entièrement reconstruite par Lefuel sous le second Empire. 
chiffre de Gabrielle d'Estrée mêlé à celui du roi qui projetait alors d'en faire son épouse $^{36}-$, évoquent les armes ou la chasse dans une frise qu'ornent également putti et animaux fabuleux et n'accordent pas de place à une quelconque figuration des arts et/ou des Lettres. Si sur la corniche du rez-de-chaussée de la façade nord sont symboliquement représentés les arts ou l'agriculture mêlés au chiffre du roi et aux regalia, elle semble plus tardive: Palma Cayet peut en effet écrire en 1605:

Tous ces bastimens par le dehors sont decorez de colomnes et d'enrichissements où sont representez les victoires et triomphes du Roy. ${ }^{37}$

Une affirmation si univoque - le décor ne paraît célébrer qu'un guerrier victorieux - est impossible à l'examen du premier étage de la façade sur le fleuve. En raison de l'état des finances royales qui n'en permet pas l'achèvement avant cette date, cette élévation est atteinte uniquement vers 1607-160938. L'opulent décor qui tapisse l'étage, couvert du chiffre royal à l'exclusion de tout autre - la reine Marie n'y a pas la place que la royale maîtresse avait pu avoir -, est alors très révélateur de la vision que la monarchie peut offrir d'elle-même. Les frontons profondément sculptés des hautes fenêtres, alternativement triangulaires et cintrés, les reliefs entre les pilastres qui séparent les croisées des niches avec lesquelles elles alternent, peuvent en effet se lire comme un véritable programme de gouvernement royal. D'est en ouest, douze travées évoquent 1) la musique, 2) les travaux des champs et l'élevage, 3) la marine commerciale, 4) à nouveau les travaux de la campagne, 5) les arts visuels, les Lettres et les sciences, 6) la chasse, 7) les armes, 8) à nouveau les arts et les sciences, 9) la marine de guerre; les travées 10 et 11 célèbrent les armes, la douzième la chasse. De part et d'autre de cet ensemble, deux panneaux surnuméraires évoquent à l'est les armes et à l'ouest les vendanges. Deux frontons triangulaires couronnent les fenêtres du pavillon ouest: ils célèbrent respectivement l'agriculture et le commerce maritime, la musique et le théâtre ${ }^{39}$. Si les armes dominent cet ensemble, si la présence d'une figuration des arts peut en partie se comprendre par l'affectation de l'étage inférieur du bâtiment à des artistes - mais non à des hommes de Lettres - que le prince soutient personnellement ${ }^{40}$, un tel parti décoratif donne bien du règne l'image non exclusivement guerrière que quelques rares et intuitifs éloges du roi écrits alors retiennent. Son sens profond pour la royauté reste difficile à évaluer: on ne sait en effet pas qui préside à cette iconographie; on ne sait pas si le roi participe à son élaboration. Mais - et là est sans aucun doute le seul point qui compte - ce décor est accepté par le souverain sur la façade d'un bâtiment à la construction duquel il a été très attaché ${ }^{41}$.

(36) Voir J.-P. BABELON, Architecture et emblématique dans les médailles de Henri IV, «Revue de l'art», n. 58/59 [1983], p. 33.

(37) P. CAYET, Chronologie septenaire de l'bistoire

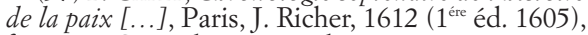
f. $448 v^{\circ}$. Cette décoration dont on ne sait exactement quand elle fut achevée - peut-elle l'être au moment où Cayet écrit? - est encore en partie visible sur la Cour Visconti. Des clichés pris avant les travaux commandés par Napoléon III en conservent également la trace; voir J.-P. BABELON, Les photographies des estampages de la Grande Galerie du Louvre avant sa restauration, «Revue de l'art», n. 58/59 [1983], pp. 46-47.

(38) Le 3 octobre 1608, Malherbe écrit à Peiresc: «Le pavillon du bout de la galerie est presque achevé; la galerie du pavillon au bâtiment des Tuile- ries est fort avancée; les fenêtres de l'étage de bas sont faites» («Lettres à Peiresc», Euvres, éd. Ant. ADAm, Paris, Gallimard, 1971, p. 410).

(39) Voir J.-P. BABELON, Les photographies des estampages..., p. 52. Le pavillon est, pour sa part, a été détruit lors de la réfection effectuée par Le Vau en 1661. Le pavillon central de la partie est de la Galerie tel qu'on peut le voir aujourd'hui, malgré sa profonde cohérence iconographique avec le reste de la façade, n'existe pas au XVII ${ }^{\mathrm{e}}$ siècle.

(40) Par lettres patentes du 22 décembre 1608, Henri IV loge au rez-de-chaussée et à l'entresol de la Galerie des artistes qu'il affranchit de la tutelle des corporations; son intention est connue au moins depuis 1605 puisque Cayet la mentionne (Loc. cit.).

(41) Voir P. Dupont, «Le Louvre et les Tuile- 
En proposant symboliquement une image du royaume florissant et pacifié, la façade de la Galerie donne à voir une sorte de portrait du roi qui a rendu possible un tel état, peut-être même une image des actions du roi tout aussi frappante qu'imprécise dans ses manifestations concrètes. Le nouvel espace de mise en scène symbolique autant que l'absence de la figure de la reine Marie donnent à l'image du roi une nouvelle ampleur - il s'agirait d'une image individuelle - et à la royauté un nouveau visage officiel - il s'agirait d'une image de l'institution monarchique qui s'incarne en son prince.

L'absence de la figure de Marie de Médicis retient ici l'attention car l'émergence d'une image nouvelle de protection royale des arts et des Lettres s'accompagne de l'effacement de l'image de la reine. Or c'est à la souveraine nouvellement arrivée en France au lendemain de son union avec le roi - nous sommes alors en 1600 - que les somptueuses cérémonies municipales avaient en quelque sorte confié le pôle culturel de la nouvelle dynastie. Héritière d'une famille célèbre pour un mécénat incarné dans la figure progressivement devenue princière de Laurent le Magnifique ${ }^{42}$, la nouvelle reine de France suscite des espoirs que son époux n'avait pas véritablement fait naître.

Que peuvent attendre les Muses, et les hommes doctes de France de cette princesse extraicte de ce docte sang que toute faveur, et secours pour fleurir plus que jamais en ce royaume, ${ }^{43}$

s'exclame André Valladier dans le livret de l'entrée de la reine à Avignon ${ }^{44}$. Un tel enthousiasme ne s'associe jamais à la figure du roi. Les «sciences» escortent la reine et, d'Italie, l'accompagnent vers la France - manière de faire une large parenthèse sur les images dynastiques du siècle précédent -; elles sont strictement attachées à sa figure et non à celle du roi à qui elle s'unit. Marie hérite, pour le plus grand bien de la France, d'une prestigieuse propension familiale à protéger les Lettres et à distinguer les lettrés.

Cette image de la reine est mise en scène sous forme d'un portrait allégorique que grave Jacques de Fornazeris et qui illustre en 1605 l'édition de L'Histoire de France et des choses memorables advenues aux provinces estrangères durant sept années de paix du regne de Henry IIII de Pierre Matthieu ${ }^{45}$. Au moment où la paix est rétablie dans le royaume de France et où l'union royale fructueuse a effectivement assuré le trône, cette gravure dit quelle place occupe la protection des Lettres dans la constitution de l'image du prince. La figure représente la France sous les traits de la reine: elle trône sous une grande couronne que supportent deux colonnes, possible symbole de la domination impériale ${ }^{46}$; dans sa main droite elle tient une épée entourée de feuillage - signe de paix -, son avant-bras gauche soutient une corne d'abon-

ries», Henri IV et la reconstruction du royaume, Paris, Réunion des musées nationaux, 1989, p. 351; G. Bresc-BAutier, Le Louvre, une histoire de Palais, Paris, Musée du Louvre-Somogy, 2008, pp. 42-46.

(42) Voir Lorenzo dopo Lorenzo. La fortuna storica di Lorenzo il Magnifico, éd. P. PIROLO, s. 1., Silvana Editoriale, 1992.

(43) A. Valladier, Labyrinthe royal de l'Hercule gaulois triomphant [...], Avignon, J. Bramereau, 1601, pp. 198-199.

(44) Lidée qu'existe une manière de nouvelle translatio d'Italie vers la France se lit aussi dans le livret qui accompagne l'entrée de la reine à Lyon; voir L'entrée de la Reine Marie de Medicis à Lyon le III Decembre 1600, Lyon, T. Ancelin, [1601], p. 15.

(45) P. Matthieu, L'Histoire de France et des choses memorables advenues aux provinces estrangeres durant sept années de paix du regne de Henry IIII, Paris, J. Mettayer, 1605; voir F. BARDON, Le portrait mythologique à la cour de France, p. 174.

(46) Sur ce thème, voir A. HARAN, Le Lys et le globe. Messianisme dynastique et rêve impérial en France aux XVI et XVII siècle, Seyssel, Champ Vallon, 2000, p. 217 et suiv. 
dance - signe de prospérité -; placé sur le socle du trône, un cartouche transcrit les paroles de la France: «Après tant de sang et de larmes, / Je suis heureuse desormais / La guerre est morte par mes armes / Mes armes font vivre la paix ${ }^{47}{ }^{\prime}$. Le paysage qui forme l'arrière-plan de la gravure peint les activités d'un peuple rendu à la paix: chasse, commerce, agriculture, réunions amicales. Mais cette gravure veut avant tout témoigner du renouveau culturel que la paix rend possible - tout en soulignant que les Lettres ne sont pas causes de la paix retrouvée -: de part et d'autre du siège royal, au premier plan, des amas importants de livres, d'instruments de musique et scientifiques occupent le regard, image paradoxale lorsque l'on sait que l'ouvrage ne s'attache en rien à cet aspect de la paix. La France voit ainsi s'épanouir, à proximité du trône, les arts et les Lettres, mais ce n'est pas sous le signe de la figure du roi. L'héritage médicéen que la reine se voit attribuer lors de ses entrées municipales trouve ici encore à s'illustrer tandis que le changement dynastique semble nécessiter un nouvel ancrage culturel de la monarchie. Jusque vers 1607, le roi, non qu'il soit indifférent au monde des Lettres, n'engage pas directement son image dans sa protection. La configuration symbolique du frontispice de 1605 ne signifie en rien que l'image de la reine lui impose de se couler dans une fonction de protection des Lettres dont elle déchargerait très officiellement le roi; elle montre que le prince prend, aux yeux de ceux qui le peignent, certaines distances avec une image humaniste majeure qui, par le biais de la reine, peut encore demeurer une image royale ${ }^{48}$. La vie des Lettres ne passe plus par une image d'un roi amateur et protecteur; la protection suffit, quelque distante qu'elle puisse être, même aux yeux des lettrés. En 1605, le frontispice de L'Histoire de France de Pierre Matthieu ne délivrait peut-être pas fondamentalement d'autre message que celui délivré par les façades de la Galerie du Louvre; mais la figuration du royaume ayant retrouvé quiétude et prospérité s'organisait alors autour d'un portrait allégorique de la reine enfermé dans les pages d'un livre; en 1609, c'est sur la façade du palais d'un roi victorieux devenu roi de paix que ces signes se lisent et dans les mois qui vont être les derniers du règne, c'est l'image du roi qui assume elle-même une fonction intégrée aux fonctions affirmées de la royauté: là est l'absolue nouveauté.

Quelques éloges du roi publiés dans les dernières années du règne enregistrent ces évolutions et témoignent d'une fine perception des changements que connaît l'image royale. Ils disent quelle orientation est en train de prendre la relation des Lettres et du prince, partant, quelle image renouvelée du prince est envisageable au cœur de cette relation. En 1609, l'anonyme Abregé de la vie de Henry Auguste quatriesme du nom proclame:

(47) Ce discours exclut l'interprétation proposée par F. A. YATEs (Astrée. Le symbolisme impérial au $X V I^{e}$ siècle, s. l., Belin, 1989, pp. 402-403) qui voit dans cette allégorie féminine une figure d'Astraea; outre le fait que la figure ne porte pas, ainsi que l'indique Yates afin de confirmer son identification, une couronne d'épis, son discours mentionne très fermement ses armes; il ne semble donc pouvoir s'agir que de la France, dans toute sa légitimité dynastique, pacifiée et rendue à la prospérité. Les vers de cette gravure peuvent se lire en écho à ceux que Malherbe écrit en 1600 pour accueillir la reine à Aix; voir «A la reine sur sa bienvenue en France», Euvres, pp. 32-38, vv. 91-95.

(48) Nous ne pouvons aborder dans ces pages un autre élément essentiel de la protection royale des Lettres, mais à partir de 1605 seulement: le retour à Paris de la reine Marguerite qui assure à sa manière le lien avec l'héritage des Valois; C. Garnier écrit à cette occasion: «Enfin, la sage, docte et belle Marguerite, / Le fleuron des Valois qui t'aimerent si fort / Comme un riche navire a surgi dans le port / De ses nobles ayeulx. / [...] / Apollon toutesfois, et les neuf Muses belles / En monstroient à l'ecart mille joyes nouvelles. / [...] / Ils jugoient qu'ils voyoient leurs puissances accreues, / Puisque les MEDICIS et que ceux de VALOIS / Se rejoignoient ensemble encore une autre fois, / Noble sang destiné pour l'heur et pour la gloire» (La Reception de la Reyne Marguerite par leurs Majestez, Paris, F. Huby, 1605, pp. 4-5). Les espoirs limités placés en Henri IV luimême se disent par le silence qui entoure son nom dans ces vers enthousiastes: l'hérédité n'est pas en sa faveur, mais parle pour Marie autant que pour Marguerite. Sur cette dernière, voir S. RAtEL, La Cour de la reine Marguerite, «Revue du XVI siècle», 11 [1924], pp. 1-24, 193-207; 12 [1925], pp. 1-43. 
Il fait soubs son heureux Empire fleurir les Lettres et les Loix, restablir partout le commerce et augmenter les arts et mestiers. ${ }^{49}$

La parcimonie de ces remarques ne doit pas voiler leur grande pertinence. La faveur envers les Lettres et les arts a sa place dans le portrait du roi, quelque mesurée, voire marginale, qu'elle soit. Noyée parmi les autres actions qui font que le roi est un bon roi, la protection des Lettres est cependant devenue un des devoirs multiples d'un roi tandis que l'image majeure demeure celle d'un roi de guerre victorieux qui œuvre au bien de ses sujets. Peut-être est-il possible, ainsi que le fait Jean Héroard, de célébrer le rétablissement de «l'honneur des bonnes lettres sur le throsne Royal, leur estime à la Cour, et par toute la Frances ${ }^{50}$; une telle célébration n'a néanmoins pas pour objet de promouvoir la constitution d'une image royale centrée sur la protection des Lettres, mais entend souligner la qualité de l'éducation du Dauphin Louis.

Au lendemain de la mort du souverain, les éloges qui retiendront la composante nouvelle du portrait royal ne seront pas de taille à forger du défunt une image de «Padre delle buone lettere» ${ }^{51}$. Les histoires du règne donneront aux Lettres et à leur protection ce qui est peut-être devenu leur juste place ${ }^{52}$ tandis qu'il est possible, sans flagornerie, quoique qu'au gré d'une manipulation des termes - les mêmes mots ne signifient plus la même chose qu'au siècle précédent -, d'inscrire Henri IV dans la lignée royale et nationale des protecteurs des hommes de Lettres ${ }^{53}$. Cependant, le pouvoir non plus que les lettrés français n'œuvrent pour qu'une telle image se déploie inconsidérément. Sur le Parnaso de Boccalini, il est possible de s'étonner que le roi ne soit pas davantage loué pour le soutien qu'il a accordé aux Lettres; mais en France les choses sont visiblement perçues autrement, au gré des modalités nouvelles de relations entre le prince souverain et les Lettres.

Il faut d'une part compter avec l'évolution des formes du gouvernement. La prise de conscience par le roi du rôle que les Lettres jouent pour fabriquer son image apparaît dans une des mentions de l'attention portée par Henri IV au monde des Lettres; Jean Bertaut dresse le portrait d'un souverain conscient de l'enjeu de ses stratégiques libéralités:

Aussi n'eut-il jamais cognoissance d'aucun excellent personnage de son Royaume, et surtout recommandé pour la gloire des lettres, qu'il ne le favorisast de quelque honneste pension; principalement s'il avoit une plume, qui peust eternellement faire vivre sa renommée, et l'honneur de ses gestes. Car comme il aymoit à faire choses louables, aussi certes aymoit-il d'estre loué.

(49) Abregé de la vie de Henry Auguste quatriesme du nom très victorieux et invincible Roy de France et de Navarre, Paris, R. Estienne, 1609; ce texte se lit aussi sous le titre Sommaire de la vie de Henry Auguste quatriesme du nom à la suite des Paralleles de Cesar et d'Henry IV par Ant. de Bandole, Paris, J. Richer, 1609, p. 128; cf. P. MatThIEU, Histoire de Louys XI, pp. 557-558.

(50) J. HÉROARD, De l'institution du prince, Paris, J. Jannon, 1609, f. $38 \mathrm{v}^{\circ}$.

(51) Voir, outre Bénévent mentionné plus haut, J. Du BoIs-Olivier, Le Pourtraict royal de Henry le Grand [...], Paris, R. Thierry, 1610, f. 37; P. FENOUILLET, Discours funebre sur la mort de Henry le Grand, Paris, Rolin Thierry, 1610, p. 81; Le deuil de la France à la mort du grand Henry IIII, dans Les Metamorphoses d'Ovide traduittes en prose françoise, Paris, Vve L'Angelier, 1617, pp. 64 et 68.

(52) Voir par ex. Les Chroniques et annales de
France [...] augmentées et continuées depuis Charles IX jusqu'à Louis XIII par G. Chappuys, Paris, S. Chappelet, 1617, partie II, f. $139 \mathrm{r}^{\circ}$. Autres témoignages qui rendent compte de la place qu'occupe la protection des Lettres dans les affaires du royaume sur l'«Inscription faite [par G. Du Vair] à l'honneur du feu Roy Henry le Grand» (Les CEuvres, Paris, S. Cramoisy, 1641, pp. 668-669) ou dans les décors commandés par P. Brûlart pour son château de Berny (Voir J. Wilhelm, Un décor disparu: les peintures du château de Berny illustrant la vie d'Henri IV et la première année du règne de Louis XIII, «Bulletin de la Société d'Histoire de l'Art Français», 1983, pp. 29-45).

(53) Voir G. Marino, L'Adone, Paris, O. de Varan, 1623, f. àijv ${ }^{\circ}$ ou G. Naudé, Addition à l'bistoire de Louys [XI], Paris, F. Targa, 1630, pp. 377-378. Une telle perspective était impensable dans les années 1590 . 
Carmen amat quisquis carmine digna facit.

C'est pourquoi des plumes d'or qu'il cognoissoit, la condition n'estoit point autre que dorée; ny jamais l'Université de Paris n'eut de si justes subjects d'esperer la resurrection. ${ }^{54}$

Le roi se montre, en protégeant les Lettres, habile souverain, soigneux de son image: un siècle ou presque de leçon faite aux princes semble porter ses fruits et Henri IV cherche à associer la gloire des Lettres qui existe en dehors de lui mais qui est connue de lui à la gloire du trône qu'il détient seul. Le prince, sensible à la notoriété d'un lettré, est en quête d'une image de soi que seules les Lettres peuvent lui apporter: échange de bons procédés avec des lettrés qui, pour certains au moins, attendent explicitement sa 'douce faveur' et sa 'grace'; le critère discriminant majeur retenu par le roi sera celui de la gloire déjà acquise par l'homme de Lettres. Quant à l'amour des Lettres, fondement humaniste de leur soutien par le prince, il n'apparait que dans le vers de Claudien; l'amour de soi, la fine perception des modalités pratiques de constitution de son image l'emporte largement sur un amour libéral de la culture lettrée. Bertaut prête au roi une intention qui répond, mais en la moralisant, à une évolution globale qui tend à faire de la protection des Lettres un possible instrument du pouvoir. La continuité éthique de l'action royale est en effet assurée par un égal amour de bien faire et de sa propre gloire; les Lettres se trouvent à la jonction de ces deux pôles tandis que l'intérêt du roi est dégagé des soupçons d'une basse manipulation. Un tel intérêt répond à la grandeur propre des Lettres. Lorsque Claude Garnier offre au jeune Louis XIII son Mausolée du grand Roy, il exhorte:

Defendez, preservez la barque des neuf Sœurs,

Qui rame entre l'effort des brigans ravisseurs,

Des pirates sans foy, leur grandeur vous en prie.

Les Muses et les Roys ont de la Symmetrie,

Ne les refusez pas. ${ }^{55}$

La grandeur des Lettres est devenue un argument pour demander à un roi son soutien; se dessinent, fût-ce faiblement, les contours d'un univers où les Lettres, quoique désarmées, auraient leur pouvoir propre.

Le règne d'Henri IV, généralement considéré comme peu favorable aux lettrés - l'image du roi au moins suggérée à partir des années 1630 est celle d'un prince assez indifférent au monde des Lettres -, marque en fait une étape majeure dans l'image royale de protection des Lettres et dans les conditions mêmes d'existence des Lettres à proximité du trône. Mutation des Lettres, mutation des images et des pratiques du pouvoir se combinent: la place prestigieuse des Lettres dans le royaume est chose acquise tandis que le lien entre les Lettres et le roi semble, sous le signe du prestige des Lettres autant que sous le signe des devoirs royaux, repositionné. La nature du lien est radicalement autre que ce qu'envisageait Budé lorsqu'il engageait François $1^{\text {er }}$ à soutenir ses entreprises: l'éventuelle collaboration du prince et des Lettres n'est plus à l'ordre du jour; on n'attend plus du prince, dans l'ordre politique qui lui est le sien, qu'il manifeste un amour particulier pour les Lettres, ni qu'il soit l'Apollon d'un quelconque Parnasse - l'image d'Hercule musagète parfois utilisée

(54) J. J. Bertaut, Oraison funèbre d'Henri IV, dans G. Du Peyrat, Les Oraisons et discours funebres de divers autheurs sur le trespas de Henry le Grand, Paris, R. Estienne et P. Chevalier, 1611, p. 69; le vers latin est un vers de Claudien, De
Consulatu Stilichonis, III, xxiii, 6, cité par Pétrarque dans son Discours du couronnement (avec gerit à la place de facit).

(55) C. Garnier, Mausolée du grand Roy [...], Paris, J. de Bordeaulx, 1611, p. 68. 
pour Henri IV est dans ce contexte véritablement significative car elle signale un échange et non une moindre fusion des domaines de chacun. Le roi peut ne considérer les Lettres que comme un élément de son royaume dont il lui convient de favoriser l'épanouissement, au même titre cependant qu'il favorise un commerce florissant - la manière d'ailleurs dont renaissance du commerce et renaissance des Lettres après les guerres civiles sont fréquemment associées dans la célébration du roi par les lettrés dit bien qu'eux-mêmes n'y voient aucune objection. Ce sont ainsi les fondements de la possible relation qui se sont transformés. Un idéal nouveau voit sans doute alors le jour et l'assassinat d'Henri IV interrompt pour un temps une évolution qui ne trouvera d'accomplissement, encore que dans une configuration bien différente, que dans les premières années du règne de Louis XIV. 